\title{
Dynamical symmetry breaking in non-Abelian geometrodynamics
}

\author{
Alcides Garat ${ }^{1}$ \\ 1. Instituto de Física, Facultad de Ciencias, Iguá 4225, esq. Mataojo, Montevideo, \\ Uruguay.
}

(Dated: September 7th, 2011)

We are going to analyze through a first order perturbative formulation the local loss of symmetry when a source of non-Abelian Yang-Mills and gravitational fields interacts with an external agent that perturbes the original geometry associated to the source. Then, as the symmetry in Abelian and non-Abelian field structures in four-dimensional Lorentzian spacetimes is displayed through the existence of local orthogonal planes of symmetry that we previously called blades one and two, the loss of symmetry will be manifested by the tilting of these planes under the influence of the external agent. It was found already that there is an algorithm to block diagonalize the Yang-Mills field strength isospace projections in a local gauge invariant way. Independently, it was also found an algorithm to diagonalize the Yang-Mills stress-energy tensor in a gauge invariant way. Using these results and perturbative analysis from a previous manuscript dealing with the Abelian case, we are going to demonstrate how to develop an algorithm for constructing local conserved currents inside both local orthogonal planes. As the interaction proceeds, the planes are going to tilt perturbatively, and in this strict sense the original local symmetries will be lost. But we will prove that the new blades at the same point will correspond after the tilting generated by perturbation, to new symmetries, with associated new local currents, both on each new local planes of symmetry. Old symmetries will be broken, new will arise. There will be a local symmetry evolution in the non-Abelian case as well. 


\section{INTRODUCTION}

The standard mechanisms for mass generation are dynamic symmetry breaking $\frac{1-11}{-1}$ and the Higgs mechanism ${ }^{12}$. Mass generated as a result of different forms of breaking symmetries. In these works Quantum Field Theoretical techniques were used. In this manuscript we will address dynamical symmetry breaking under the scope of a classical and geometrical point of view. We have to be clear about the goals of this note, we are not aiming at mass generation, but the generation of a change in curvature that will be responsible for the symmetry breaking. We are assuming the existence of classical sources of gravitational fields where in addition the sources generate non-Abelian Yang-Mills fields. It is then appropriate to ask for the relationship between our study and the works cited above. For this purpose

we have to review the results found in a previous work like manuscript ${ }^{13}$. In this paper we found that locally the electromagnetic gauge group of transformations is isomorphic to tetrad Lorentz transformations in both orthogonal planes or blades, one and two $\underline{14}$. That is to say, isomorphic to local Lorentz transformations on both planes, independently. It was found that at every point in a curved four-dimensional Lorentzian spacetime where a non-null electromagnetic field is present, a tetrad can be built such that these vectors covariantly diagonalize the electromagnetic stress-energy tensor at every point in spacetime. Let us display for the Abelian case the explicit expression for these vectors,

$$
\begin{aligned}
U^{\alpha} & =\xi^{\alpha \lambda} \xi_{\rho \lambda} A^{\rho} /\left(\sqrt{-Q / 2} \sqrt{A_{\mu} \xi^{\mu \sigma} \xi_{\nu \sigma} A^{\nu}}\right) \\
V^{\alpha} & =\xi^{\alpha \lambda} A_{\lambda} /\left(\sqrt{A_{\mu} \xi^{\mu \sigma} \xi_{\nu \sigma} A^{\nu}}\right) \\
Z^{\alpha} & =* \xi^{\alpha \lambda} * A_{\lambda} /\left(\sqrt{* A_{\mu} * \xi^{\mu \sigma} * \xi_{\nu \sigma} * A^{\nu}}\right) \\
W^{\alpha} & =* \xi^{\alpha \lambda} * \xi_{\rho \lambda} * A^{\rho} /\left(\sqrt{-Q / 2} \sqrt{* A_{\mu} * \xi^{\mu \sigma} * \xi_{\nu \sigma} * A^{\nu}}\right),
\end{aligned}
$$

where $Q=\xi_{\mu \nu} \xi^{\mu \nu}=-\sqrt{T_{\mu \nu} T^{\mu \nu}}$ according to equations (39) in $\frac{15}{}$. $Q$ is assumed not to be zero, because we are dealing with non-null electromagnetic fields. The four vectors (1-4) possess the following algebraic properties,

$$
-U^{\alpha} U_{\alpha}=V^{\alpha} V_{\alpha}=Z^{\alpha} Z_{\alpha}=W^{\alpha} W_{\alpha}=1
$$

We can always introduce at every point in spacetime a duality rotation by an angle $-\alpha$ that transforms a non-null electromagnetic field $f_{\mu \nu}$ into an extremal field $\xi_{\mu \nu}$, 


$$
\xi_{\mu \nu}=e^{-* \alpha} f_{\mu \nu}=\cos (\alpha) f_{\mu \nu}-\sin (\alpha) * f_{\mu \nu} .
$$

where $* f_{\mu \nu}=\frac{1}{2} \epsilon_{\mu \nu \sigma \tau} f^{\sigma \tau}$ is the dual tensor of $f_{\mu \nu}$. The extremal field satisfies the equation,

$$
\xi_{\alpha \mu} * \xi^{\mu \nu}=0
$$

which is equation (64) in $\underline{\underline{15}}$ and allows to prove the orthogonality of vectors (1-44) along with the identity,

$$
\xi_{\mu \alpha} \xi^{\nu \alpha}-* \xi_{\mu \alpha} * \xi^{\nu \alpha}=\frac{1}{2} \delta_{\mu}{ }^{\nu} Q
$$

The local scalar $\alpha$ is known as the complexion of the electromagnetic field. It is a local gauge invariant quantity and the explicit expression for the complexion results in $\tan (2 \alpha)=-f_{\mu \nu} * f^{\mu \nu} / f_{\lambda \rho} f^{\lambda \rho}$. The duality rotation given by equation (59) in $\underline{15}$,

$$
f_{\mu \nu}=\xi_{\mu \nu} \cos \alpha+* \xi_{\mu \nu} \sin \alpha,
$$

allows us to express the stress-energy tensor in terms of the extremal field,

$$
T_{\mu \nu}=\xi_{\mu \lambda} \xi_{\nu}{ }^{\lambda}+* \xi_{\mu \lambda} * \xi_{\nu}{ }^{\lambda} .
$$

At every point in a curved four-dimensional Lorentzian spacetime the new tetrad $\underline{16}, \underline{17}$ (14-4) locally and covariantly diagonalizes the stress-energy tensor (10). Written in terms of these tetrad vectors, the electromagnetic field is,

$$
f_{\alpha \beta}=-2 \sqrt{-Q / 2} \cos \alpha U_{[\alpha} V_{\beta]}+2 \sqrt{-Q / 2} \sin \alpha Z_{[\alpha} W_{\beta]} .
$$

Equation (11) entails the maximum simplification in the expression of the electromagnetic field. The true degrees of freedom are the local scalars $\sqrt{-Q / 2}$ and $\alpha$.

We remind ourselves that it was proved in manuscript $\frac{13}{3}$ that the group of local electromagnetic gauge transformations is isomorphic to the local group LB1 of boosts plus discrete transformations on blade one, and independently to LB2, the local group of rotations on blade two. The object $U_{[\alpha} V_{\beta]}$ remains invariant $\frac{13}{\underline{ }}$ under a local electromagnetic gauge transformation which is equivalent to a "rotation" or a boost of the tetrad vectors $U^{\alpha}$ and $V^{\alpha}$ by 
a scalar angle $\phi$ on blade one. This is the way in which local gauge invariance is manifested explicitly on this local plane. Analogous for discrete transformations on blade one. Similar analysis on blade two. A spatial "rotation" of the tetrad vectors $Z^{\alpha}$ and $W^{\alpha}$ through an "angle" $\varphi$ such that the object $Z_{[\alpha} W_{\beta]}$ remains invariant ${ }^{13}$. All this formalism clearly provides a technique to maximally simplify the expression for the electromagnetic field strength. It is block diagonalized automatically by the tetrad (11-4). This is not the case for the nonAbelian $S U(2)$ field strength. We do not have an automatic block diagonalization. We do not have an automatic covariant local diagonalization for the Yang-Mills $S U(2)$ stress-energy tensor either. To this purpose new algorithms were developed in references $\underline{18}, \underline{19}$. Therefore, the symmetry represented by local electromagnetic gauge transformations can be thought of as the symmetry represented by local Lorentz transformations of the tetrad unit vectors inside these blades. Blade one is generated by a timelike and a spacelike vectors, that is $\left(U^{\alpha}, V^{\alpha}\right)$. Blade two by the other two spacelike vectors $\left(Z^{\alpha}, W^{\alpha}\right)$. Subsequently it was found in manuscript ${ }^{20}$ that at every point in spacetime we can build conserved current vectors on both local planes. A whole system of perturbation analysis was introduced in order to deal with the evolution of local symmetries. In turn, a curved four-dimensional Lorentzian spacetime where non-Abelian Yang-Mills fields are present can be analyzed through the use of new local tetrads and the local planes they define that serve a multiple purpose. On one hand enable a manifest description of local gauge transformations, Abelian and nonAbelian such that these keep the metric tensor invariant. On the other hand allow for the local diagonalization of the Yang-Mills stress-energy tensor ${ }^{19}$. Additionally, it was developed in manuscript ${ }^{18}$ an algorithm to block diagonalize any Yang-Mills field strength in a local gauge invariant way. Several local isospace vectors are involved in these diagonalization algorithms. In a previous manuscript $\frac{18}{18}$ two vectors were used to block diagonalize a YangMills field strength isospace projection. In this manuscript we are going to show how to use five local isospace vectors in order to develop a different algorithm. These vectors will allow to diagonalize the Yang-Mills stress-energy tensor as in manuscript $\frac{19}{}$ and simultaneously to find local energy-momentum conserved currents on the local planes that diagonalize the stress-energy tensor. In addition they will allow for the field strength isospace projection block diagonalization. Once again we are going to choose our local planes of symmetry as the ones that diagonalize the stress-energy tensor as in the electromagnetic case. Then, we might argue the following. First, mass needs to be associated to a dynamic process of 
symmetry breaking or another process like the Higgs $\frac{12}{2}$ mechanism when addressed from the point of view of the standard model where gravitational fields are not present. Second, the very notion of symmetry breaking in the context where symmetries are treated as conserved properties that might be broken with the ensuing mass generation, phenomenon that leads to the results enumerated in the previous list of standard model approaches, is reformulated in this manuscript. In our geometrical context where local gauge transformations are reinterpreted as local Lorentz tetrad transformations, symmetries are broken by the action of external geometrical agents in the sense that the local planes of symmetry associated to the diagonalization of the Yang-Mills stress-energy tensor will be tilting as the evolution of the interaction takes place. The stress-energy tensor itself will be perturbed under the action of an external agent. Symmetries will be broken in the sense that there will be new planes or blades of diagonalization of the Yang-Mills stress-energy tensor at every point such that new symmetries will arise as time evolves. Symmetries of analogous nature but on new local planes, along with new associated local conserved currents. Symmetries then, will evolve dynamically under this new point of view. Mass within the context of the standard model is generated through dynamical symmetry breaking or the Higgs mechanism, in our context what is generated is a change in curvature. In section II we are going to introduce an algorithm to construct the new energy-momentum local currents on both planes for the non-Abelian local $S U(2)$ case. In section III the first order perturbative scheme for these field structures is introduced. Finally, in section [V] we are going to analyze the geometrical meaning of dynamical symmetry breaking for non-Abelian geometrodynamics. Throughout the paper we use the conventions of manuscript $\frac{15}{15}$. In particular we use a metric with sign conventions -+++ . The only difference in notation with $\frac{15}{5}$ will be that we will call our geometrized electromagnetic potential $A^{\alpha}$, where $f_{\mu \nu}=A_{\nu ; \mu}-A_{\mu ; \nu}$ is the geometrized electromagnetic field $f_{\mu \nu}=\left(G^{1 / 2} / c^{2}\right) F_{\mu \nu}$. Analogously, $f_{\mu \nu}^{k}$ are the geometrized Yang-Mills field components, $f_{\mu \nu}^{k}=\left(G^{1 / 2} / c^{2}\right) F_{\mu \nu}^{k}$.

\section{NEW LOCAL NON-ABELIAN CONSERVED CURRENTS}

We are going to consider for instance the Einstein-Maxwell-Yang-Mills vacuum field equations, 


$$
\begin{aligned}
R_{\mu \nu} & =T_{\mu \nu}^{(y m)}+T_{\mu \nu}^{(\mathrm{em})} \\
f_{; \nu}^{\mu \nu} & =0 \\
* f_{; \nu}^{\mu \nu} & =0 \\
f_{\mid \nu}^{k \mu \nu} & =0 \\
* f_{\mid \nu}^{k \mu \nu} & =0 .
\end{aligned}
$$

The symbol ";" stands for covariant derivative with respect to the metric tensor $g_{\mu \nu}$. The symbol | represents a gauge covariant derivative, see manuscript $\frac{19}{}$. In principle we are going to concentrate ourselves in non-Abelian fields and its perturbations as we will see in section III, but without harming our treatment we can also introduce electromagnetic fields that we emphasize are not going to be considered later on in section III to be perturbed by an external agent to the source. The field equations (13)-14) provide a hint about the existence of two electromagnetic field potentials 21 , as said in the first paper "Tetrads in geometrodynamics" reference ${ }^{13}$, not independent from each other, but due to the symmetry of the equations, available for our construction. $A^{\mu}$ and $* A^{\mu}$ are the two electromagnetic potentials ${ }^{21}$. $* A^{\mu}$ is therefore a name, we are not using the Hodge map at all in this case. Similar for the two Non-Abelian equations (15, 16). The Non-Abelian potential $A^{k \mu}$ is available for our construction as well $19, \underline{22}, \underline{23}, \underline{24}$. Following the ideas of manuscript $\frac{19}{9}$ we managed to construct a new extremal field, invariant under $S U(2) \times U(1)$ local gauge transformations, that we called $\epsilon_{\mu \nu}$ and that we can anticipate will be introduced in equation (49) for our particular problem. This new kind of local $S U(2)$ gauge invariant extremal tensor $\epsilon_{\mu \nu}$, allows in turn for the construction of the new tetrad,

$$
\begin{aligned}
& S_{(1)}^{\mu}=\epsilon^{\mu \lambda} \epsilon_{\rho \lambda} X^{\rho} \\
& S_{(2)}^{\mu}=\sqrt{-Q_{y m} / 2} \epsilon^{\mu \lambda} X_{\lambda} \\
& S_{(3)}^{\mu}=\sqrt{-Q_{y m} / 2} * \epsilon^{\mu \lambda} Y_{\lambda} \\
& S_{(4)}^{\mu}=* \epsilon^{\mu \lambda} * \epsilon_{\rho \lambda} Y^{\rho},
\end{aligned}
$$

where $Q_{y m}=\epsilon_{\mu \nu} \epsilon^{\mu \nu}$ that we assume not to be zero. The condition imposed on the $\epsilon_{\mu \nu}$ in order to be an extremal field, see reference ${ }^{19}$, is, 


$$
\epsilon_{\mu \nu} * \epsilon^{\mu \nu}=0
$$

With the help of identity,

$$
A_{\mu \alpha} B^{\nu \alpha}-* B_{\mu \alpha} * A^{\nu \alpha}=\frac{1}{2} \delta_{\mu}{ }^{\nu} A_{\alpha \beta} B^{\alpha \beta}
$$

which is valid for every pair of antisymmetric tensors in a four-dimensional Lorentzian spacetime ${ }^{15}$, we can prove that when applied to the case $A_{\mu \alpha}=\epsilon_{\mu \alpha}$ and $B^{\nu \alpha}=* \epsilon^{\nu \alpha}$ yields the equivalent condition to (21),

$$
\epsilon_{\alpha \nu} * \epsilon^{\mu \nu}=0
$$

It is straightforward using (22) for $A_{\mu \alpha}=\epsilon_{\mu \alpha}$ and $B^{\nu \alpha}=\epsilon^{\nu \alpha}$, and (23), to prove that vectors (17,20) are orthogonal. As we did before we are going to call for future reference for instance $\epsilon^{\mu \lambda} \epsilon_{\rho \lambda}$ the skeleton of the tetrad vector $S_{(1)}^{\mu}$, and $X^{\rho}$ the gauge vector. In the case of $S_{(3)}^{\mu}$, the skeleton will be $* \epsilon^{\mu \lambda}$, and $Y_{\lambda}$ will be the gauge vector. It is clear now that skeletons must be gauge invariant under $S U(2) \times U(1)$. This property guarantees that the vectors under local $U(1)$ or $S U(2)$ gauge transformations are not going to leave their original planes or blades, keeping therefore the metric tensor explicitly invariant. We have still pending the choice that we can make for the two gauge vector fields $X^{\sigma}$ and $Y^{\sigma}$ in (17, 20) such that we can reproduce in the $S U(2)$ environment, the tetrad transformation properties of the Abelian environment like shown in manuscripts $\underline{13}, \underline{19}$. The choice we are going to make is $X^{\sigma}=Y^{\sigma}=\operatorname{Tr}\left[\Sigma^{\alpha \beta} E_{\alpha}{ }^{\rho} E_{\beta}{ }^{\lambda} * \xi_{\rho}{ }^{\sigma} * \xi_{\lambda \tau} A^{\tau}\right] . A^{\tau}$ is the non-Abelian connection. The object $\Sigma^{\alpha \beta}$ is analyzed in detail in reference ${ }^{19}$, it basically translates local $S U(2)$ gauge transformations into local Lorentz spatial transformations. The tetrad $E_{\alpha}{ }^{\rho}$ will be the electromagnetic tetrad introduced in reference ${ }^{13}$, and we call $E_{(o)}^{\rho}=U^{\rho}, E_{(1)}^{\rho}=V^{\rho}, E_{(2)}^{\rho}=Z^{\rho}, E_{(3)}^{\rho}=W^{\rho}$ as in equations (1, 4). We need the electromagnetic tetrads in our construction but we are not going to perturb them in section [II since we are going to focus on perturbations to the metric tensor and the Yang-Mills field strength only. The electromagnetic extremal tensor $\xi_{\rho \sigma}$, and its dual $* \xi_{\rho \sigma}$ are also already known from reference ${ }^{13}$. The tensor structure $E_{\alpha}^{[\rho} E_{\beta}^{\lambda]} * \xi_{\rho \sigma} * \xi_{\lambda \tau}$ is invariant under $U(1)$ local gauge transformations. Essentially, because 
of the electromagnetic extremal field property $\underline{13} \underline{\underline{15}}, \xi_{\mu \sigma} * \xi^{\mu \tau}=0$. When we perform a local $U(1)$ gauge transformation of the electromagnetic tetrad vectors, it is easy to prove that the condition $\xi_{\mu \sigma} * \xi^{\mu \tau}=0$, satisfied because of the very definition of the electromagnetic extremal field, is what ensures its invariance. Next, and following the notation in reference ${ }^{19}$, we would like to introduce the tetrad $W_{(o)}^{\mu}, W_{(1)}^{\mu}, W_{(2)}^{\mu}, W_{(3)}^{\mu}$, (no confusion should arise with vector $E_{(3)}^{\rho}=W^{\rho}$ which is just one vector in the electromagnetic tetrad) which we consider to be the normalized version of $S_{(1)}^{\mu}, S_{(2)}^{\mu}, S_{(3)}^{\mu}, S_{(4)}^{\mu}$, and we perform the gauge transformations on blades one and two,

$$
\begin{aligned}
& \tilde{W}_{(o)}^{\mu}=\cosh \phi W_{(o)}^{\mu}+\sinh \phi W_{(1)}^{\mu} \\
& \tilde{W}_{(1)}^{\mu}=\sinh \phi W_{(o)}^{\mu}+\cosh \phi W_{(1)}^{\mu} \\
& \tilde{W}_{(2)}^{\mu}=\cos \psi W_{(2)}^{\mu}-\sin \psi W_{(3)}^{\mu} \\
& \tilde{W}_{(3)}^{\mu}=\sin \psi W_{(2)}^{\mu}+\cos \psi W_{(3)}^{\mu} .
\end{aligned}
$$

That equations (24,25) are the result of a local $S U(2)$ gauge transformation of the vectors $\left(W_{(o)}^{\alpha}, W_{(1)}^{\alpha}\right)$ on blade one at every point was proven in reference ${ }^{19}$. Similar for equations (26. 27) on blade two for the vectors $\left(W_{(2)}^{\alpha}, W_{(3)}^{\alpha}\right)$. It was also proven there that the local group of $S U(2)$ gauge transformations is isomorphic to the triple tensor product $(\otimes L B 1)^{3}$ and independently also to $(\otimes L B 2)^{3}$ see manuscript ${ }^{19}$. It is very easy to check that the equalities $\tilde{W}_{(o)}^{[\alpha} \tilde{W}_{(1)}^{\beta]}=W_{(o)}^{[\alpha} W_{(1)}^{\beta]}$ and $\tilde{W}_{(2)}^{[\alpha} \tilde{W}_{(3)}^{\beta]}=W_{(2)}^{[\alpha} W_{(3)}^{\beta]}$ are true. These equalities are telling us that these antisymmetric tetrad objects are gauge invariant.

\section{A. Yang-Mills field strength block diagonalization}

We briefly remind ourselves from reference $\frac{18}{}$ that we can introduce a generalized duality transformation for non-Abelian fields. For instance we might choose,

$$
\bar{\epsilon}_{\mu \nu}=\operatorname{Tr}\left[\vec{m} \cdot f_{\mu \nu}-\vec{l} \cdot * f_{\mu \nu}\right]
$$

where $f_{\mu \nu}=f_{\mu \nu}^{a} \sigma^{a}, \vec{m}=m^{a} \sigma^{a}$ and $\vec{l}=l^{a} \sigma^{a}$ are vectors in isospace. The $\cdot$ means again product in isospace. Once more we stress that $\sigma^{a}$ are the Pauli matrices (see the first appendix in reference ${ }^{19}$ ) and the summation convention is applied on the internal index $a$. The vector components are defined as, 


$$
\begin{aligned}
& \vec{m}=\left(\cos \alpha_{1}, \cos \alpha_{2}, \cos \alpha_{3}\right) \\
& \vec{l}=\left(\cos \beta_{1}, \cos \beta_{2}, \cos \beta_{3}\right),
\end{aligned}
$$

where all the six isoangles are local scalars that satisfy,

$$
\begin{aligned}
& \sum_{a=1}^{3} \cos ^{2} \alpha_{a}=1 \\
& \Sigma_{a=1}^{3} \cos ^{2} \beta_{a}=1 .
\end{aligned}
$$

In isospace $\vec{m}=m^{a} \sigma^{a}$ transforms under a local $S U(2)$ gauge transformation $S$, as $S^{-1} \vec{m} S$, see chapter III in ${ }^{25}$ and also reference ${ }^{26}$, and similar for $\vec{l}=l^{a} \sigma^{a}$. We can think this local isospace transformation as a local three-dimensional rotation $\Lambda_{b}^{a}$ in isospace, $\overrightarrow{m^{\prime}}=m^{a} \sigma^{a}=\Lambda_{b}^{a} m^{b} \sigma^{a}=S^{-1} m^{a} \sigma^{a} S=S^{-1} \vec{m} S$. The tensor $f_{\mu \nu}=f_{\mu \nu}^{a} \sigma^{a}$ transforms as $f_{\mu \nu} \rightarrow S^{-1} f_{\mu \nu} S$. Therefore $\bar{\epsilon}_{\mu \nu}$ is manifestly gauge invariant. We can see from (29, 30 ) and (31,32) that only four of the six angles in isospace are independent. It is precisely this $\bar{\epsilon}_{\mu \nu}$ that we use to locally block diagonalize a particular projection in isospace of the field strength. Because the two local unit isovectors $\vec{m}$ and $\vec{l}$ provide four local scalar variables to be fixed by the block diagonalization conditions, see $\frac{18}{}$. When we developed this method in manuscript $\frac{18}{2}$ we had the isovector $\vec{n}$ that locally projects the field strength, fixed at the outset of the procedure. In our more general system of ideas in our present manuscript, this local unit isovector $\vec{n}$ will not be fixed at the outset and will become two more local scalar variables to be found in a fashion that we explain as follows. Once again we are going to notice that $f_{\mu \nu}=f_{\mu \nu}^{a} \sigma^{a}$, and $\vec{n}=n^{a} \sigma^{a}$ are vectors in isospace. We also emphasize that by $\bar{f}_{\mu \nu}$ we mean the projection $\operatorname{Tr}\left[\vec{n} \cdot f_{\mu \nu}\right]=n^{a} f_{\mu \nu}^{a}$ where again the summation convention is applied on the internal index $a$. The vector components are defined as,

$$
\vec{n}=\left(\cos \theta_{1}, \cos \theta_{2}, \cos \theta_{3}\right)
$$

where all the three isoangles are local scalars that satisfy,

$$
\Sigma_{a=1}^{3} \cos ^{2} \theta_{a}=1
$$


In isospace $\vec{n}=n^{a} \sigma^{a}$ transforms under a local $S U(2)$ gauge transformation $S$, as $S^{-1} \vec{n} S$, see chapter III in ${ }^{25}$ and also reference ${ }^{26}$. The tensor $f_{\mu \nu}=f_{\mu \nu}^{a} \sigma^{a}$ is going to transform as $f_{\mu \nu} \rightarrow S^{-1} f_{\mu \nu} S$ as already stated above. Therefore, $\bar{f}_{\mu \nu}$ which is nothing but compact notation for $\operatorname{Tr}\left[\vec{n} \cdot f_{\mu \nu}\right]$ is a local $S U(2)$ gauge invariant object. Then, by making use of the results obtained in reference $\frac{18}{\underline{1}}$ we are going to write the block diagonalized non-Abelian field strength and its dual as,

$$
\begin{gathered}
\bar{f}_{\mu \nu}=A \bar{\epsilon}_{\mu \nu}+B * \bar{\epsilon}_{\mu \nu} \\
* \bar{f}_{\mu \nu}=-B \bar{\epsilon}_{\mu \nu}+A * \bar{\epsilon}_{\mu \nu},
\end{gathered}
$$

where $A$ and $B$ are local scalars. The extremal field tensor and its dual that correspond to this particular block diagonalized field strength (35), see expression (28), can then be written,

$$
\begin{aligned}
\bar{\epsilon}_{\mu \nu} & =-2 \sqrt{-Q_{y m} / 2} \bar{T}_{(o)[\mu} \bar{T}_{(1) \nu]} \\
* \bar{\epsilon}_{\mu \nu} & =2 \sqrt{-Q_{y m} / 2} \bar{T}_{(2)[\mu} \bar{T}_{(3) \nu]} .
\end{aligned}
$$

Let us remember that from all the possible tetrads introduced above $W_{(o)}^{\mu}, W_{(1)}^{\mu}, W_{(2)}^{\mu}$, $W_{(3)}^{\mu}$, in reference $\frac{18}{2}$ we designated $\bar{T}_{(o)}^{\mu}, \bar{T}_{(1)}^{\mu}, \bar{T}_{(2)}^{\mu}, \bar{T}_{(3)}^{\mu}$ the particular tetrad that locally block diagonalizes the field strength $\bar{f}_{\mu \nu}$. Equations (37) 38) are providing the necessary information to express the non-Abelian field strength projection by $\vec{n}$ in terms of the new tetrad,

$$
\bar{f}_{\mu \nu}=-2 \sqrt{-Q_{y m} / 2} A \bar{T}_{(o)[\mu} \bar{T}_{(1) \nu]}+2 \sqrt{-Q_{y m} / 2} B \bar{T}_{(2)[\mu} \bar{T}_{(3) \nu]} .
$$

Next, using equation $\bar{\epsilon}_{\alpha \nu} * \bar{\epsilon}^{\mu \nu}=0$ for the extremal field that corresponds to local block diagonalization as analyzed in reference ${ }^{18}$ and also equations (28) and (35) we can write the local scalars $A$ and $B$ as follows,

$$
\begin{aligned}
& A=\bar{f}_{\mu \nu} \bar{\epsilon}^{\mu \nu} / \bar{\epsilon}_{\sigma \tau} \bar{\epsilon}^{\sigma \tau} \\
& B=\bar{f}_{\mu \nu} * \bar{\epsilon}^{\mu \nu} / * \bar{\epsilon}_{\sigma \tau} * \bar{\epsilon}^{\sigma \tau} .
\end{aligned}
$$


We can also write the extremal and its dual as,

$$
\begin{aligned}
\bar{\epsilon}_{\mu \nu} & =\frac{A}{\left(A^{2}+B^{2}\right)} \bar{f}_{\mu \nu}-\frac{B}{\left(A^{2}+B^{2}\right)} * \bar{f}_{\mu \nu} \\
* \bar{\epsilon}_{\mu \nu} & =\frac{B}{\left(A^{2}+B^{2}\right)} \bar{f}_{\mu \nu}+\frac{A}{\left(A^{2}+B^{2}\right)} * \bar{f}_{\mu \nu} .
\end{aligned}
$$

It is very important at this point to remind ourselves that the isovector (33) satisfying the condition (34) was to be determined in a complete independent way with respect to the local block diagonalization process. It was claimed in reference ${ }^{18}$ that this isovector (33) was given at the outset of the block diagonalization process. We developed in manuscript $\frac{18}{18}$ a method to block diagonalize in a gauge invariant way, isospace projections of the nonAbelian field strength by this isovector (33). Now, imagine that within this new context we do not provide this isovector at the outset of the algorithm. On the contrary, its two local independent variables $\left(\cos \theta_{1}, \cos \theta_{2}\right)\left(\cos \theta_{3}\right.$ is found through the normalizing condition (34) ) become now two variables available for our new system of equations. The system of equations that determines locally the two new conserved currents that live or lie inside blades one and two.

\section{B. Yang-Mills stress-energy diagonalization}

It is important to remind ourselves and review the local gauge invariant algorithm for the diagonalization of the Yang-Mills stress-energy tensor. This process which we emphasize to be completely independent of the algorithm for the block diagonalization of the YangMills field strength isospace projections. We start by introducing a new generalized duality transformation for non-Abelian fields as we did in section \A,

$$
\tau_{\mu \nu}=\operatorname{Tr}\left[\vec{p} \cdot f_{\mu \nu}-\vec{q} \cdot * f_{\mu \nu}\right]
$$

where $f_{\mu \nu}=f_{\mu \nu}^{a} \sigma^{a}, \vec{p}=p^{a} \sigma^{a}$ and $\vec{q}=q^{a} \sigma^{a}$ are vectors in isospace. The $\cdot$ means product in isospace. $\sigma^{a}$ are the pauli matrices, see the first appendix in reference $\frac{19}{}$, and the summation convention is applied on the internal index $a$. The vector components are defined as, 


$$
\begin{aligned}
& \vec{p}=\left(\cos \delta_{1}, \cos \delta_{2}, \cos \delta_{3}\right) \\
& \vec{q}=\left(\cos \gamma_{1}, \cos \gamma_{2}, \cos \gamma_{3}\right),
\end{aligned}
$$

where all the six isoangles are local scalars that satisfy,

$$
\begin{aligned}
& \Sigma_{a=1}^{3} \cos ^{2} \delta_{a}=1 \\
& \Sigma_{a=1}^{3} \cos ^{2} \gamma_{a}=1 .
\end{aligned}
$$

In isospace $\vec{p}=p^{a} \sigma^{a}$ transforms under a local $S U(2)$ gauge transformation $S$, as $S^{-1} \vec{p} S$, see chapter III in ${ }^{25}$ and also reference $\underline{26}$, and similar for $\vec{q}=q^{a} \sigma^{a}$. The tensor $f_{\mu \nu}=f_{\mu \nu}^{a} \sigma^{a}$ transforms as $f_{\mu \nu} \rightarrow S^{-1} f_{\mu \nu} S$. Therefore $\tau_{\mu \nu}$ is manifestly gauge invariant. We can see from (45-46) and (47,48) that only four of the six angles in isospace are independent. Next we perform one more duality transformation,

$$
\epsilon_{\mu \nu}=\cos \alpha_{d} \tau_{\mu \nu}-\sin \alpha_{d} * \tau_{\mu \nu}
$$

such that the complexion $\alpha_{d}$ is defined by the usual local condition $\epsilon_{\mu \nu} * \epsilon^{\mu \nu}=0$, see reference $^{13}$,

$$
\tan \left(2 \alpha_{d}\right)=-\tau_{\mu \nu} * \tau^{\mu \nu} / \tau_{\lambda \rho} \tau^{\lambda \rho}
$$

All the conclusions derived in $\frac{13}{}$ are valid in this context and therefore exactly as in reference ${ }^{13}$. Using the local antisymmetric tensor $\epsilon_{\mu \nu}$, we can produce tetrad skeletons and with new gauge vectors $X_{d}^{\sigma}$ and $Y_{d}^{\sigma}$ we can build a new normalized tetrad like the normalized version of (17-20). In the end, it is a particular version of the tetrad $W_{(o)}^{\mu}, W_{(1)}^{\mu}, W_{(2)}^{\mu}, W_{(3)}^{\mu}$, this time the tetrad that locally diagonalizes the Yang-Mills stress-energy tensor. This new tetrad that we call $T_{\alpha}^{\mu}$ has four independent isoangles included in its definition, in the skeletons. There is also the freedom to introduce an LB1 and an LB2 local $S U(2)$ generated transformations on both blades by new angles $\phi_{d}$ and $\psi_{d}$ (through the gauge vectors $X_{d}^{\sigma}$ and $Y_{d}^{\sigma}$ ) which are not yet fixed and represent two more independent angles. Having six independent and undefined angles, we are going to use this freedom to choose them when 
fixing the six diagonalization conditions for the stress-energy tensor. It must be highlighted and stressed that since the local antisymmetric tensor $\epsilon_{\mu \nu}$ is gauge invariant, then the tetrad vectors skeletons are $S U(2)$ gauge invariant. This was a fundamental condition that we made in previous sections in order to ensure the metric invariance when performing LB1 and LB2 transformations. Then, we proceed to impose the diagonalization conditions,

$$
\begin{aligned}
& T_{o 1}=T_{o}^{\mu} T_{\mu \nu} T_{1}^{\nu}=0 \\
& T_{o 2}=T_{o}^{\mu} T_{\mu \nu} T_{2}^{\nu}=0 \\
& T_{o 3}=T_{o}^{\mu} T_{\mu \nu} T_{3}^{\nu}=0 \\
& T_{12}=T_{1}^{\mu} T_{\mu \nu} T_{2}^{\nu}=0 \\
& T_{13}=T_{1}^{\mu} T_{\mu \nu} T_{3}^{\nu}=0 \\
& T_{23}=T_{2}^{\mu} T_{\mu \nu} T_{3}^{\nu}=0 .
\end{aligned}
$$

These are finally the six equations that locally define the six angles $\delta_{1}, \delta_{2}, \gamma_{1}, \gamma_{2}, \phi_{d}, \psi_{d}$, for instance. The other two $\delta_{3}, \gamma_{3}$ are determined by equations (47,48) once the other six have already been determined through equations (51-56). We imposed the off-diagonal tetrad components of the stress-energy tensor (52,55) to be zero. These four equations are manifestly and locally $S U(2)$ gauge invariant by themselves under LB1 and LB2 local transformations of the vectors $T_{\alpha}^{\mu}$, analogous to transformations (24)27). We would be able to write the diagonalized expression for the stress-energy tensor as,

$$
T_{\mu \nu}=\left[-C T_{(o) \mu} T_{(o) \nu}+D T_{(1) \mu} T_{(1) \nu}+E T_{(2) \mu} T_{(2) \nu}+F T_{(3) \mu} T_{(3) \nu}\right],
$$

where $C, D, E, F$ are local scalars. We must stress that the stress-energy tensor either in equations (51,56) or equation (57) is $T_{\mu \nu}=T_{\mu \nu}^{(y m)}+T_{\mu \nu}^{(e m)}$, but we could have chosen only $T_{\mu \nu}=T_{\mu \nu}^{(y m)}$ as well. It is evident that we are assuming $T_{(o)}^{\mu}$ to be the timelike vector. It is evident that the "diagonal gauge" will be a source of simplification in dealing with perturbative analysis, and of course the inherent simplification in the geometrical analysis of any problem involving these kind of fields (12, 16). 


\section{New conserved energy-momentum local currents}

Having putting forward all the necessary elements in previous sections, we take on the task of imposing two equations for the local energy-momentum currents,

$$
\begin{aligned}
\left(T_{\tau \rho} * \epsilon^{\rho \nu} B_{\nu}\right. & \left.g^{\tau \mu}\right)_{; \mu}=0 \\
\left(T_{\tau \rho} \epsilon^{\rho \nu} B_{\nu}\right. & \left.g^{\tau \mu}\right)_{; \mu}=0 .
\end{aligned}
$$

We use the notation $B_{\nu}=B_{, \nu}$ for short. These two equations (58,59) represent two equations for the two remaining local unknowns $\left(\cos \theta_{1}, \cos \theta_{2}\right)$ defined in equation (33). What we have in the end are the four conditions imposed in order to block diagonalize the field strength in a local gauge invariant way, plus equations (58,59), making up six equations for six local scalars ( $\left.\cos \alpha_{1}, \cos \alpha_{2}, \cos \beta_{1}, \cos \beta_{2}, \cos \theta_{1}, \cos \theta_{2}\right)$. The stress-energy tensor $T_{\mu \nu}$ is already diagonal such that the skeletons of the local tetrad $T_{\alpha}^{\mu}$ that diagonalizes this YangMills stress-energy tensor are built using the $\epsilon^{\mu \nu}$ as found in section IIB. Let us remember that in the expression $\bar{f}_{\mu \nu}=\operatorname{Tr}\left[\vec{n} \cdot f_{\mu \nu}\right]$ the isovector (33) is included, and therefore, it is included in the local scalars $A$ and $B$ through (40,41). It is also apparent that the local vector $\epsilon^{\mu \nu} B_{\nu}$ lies on the local blade one that corresponds to the local diagonalization of the Yang-Mills stress-energy tensor, and the local vector $* \epsilon^{\mu \nu} B_{\nu}$ lies on the local blade two for the same diagonalization algorithm, for this purpose see the tetrad (17,-20) and the condition (23) along with the assumption that the tetrad $T_{(o)}^{\mu}, T_{(1)}^{\mu}, T_{(2)}^{\mu}, T_{(3)}^{\mu}$ is the normalized version of the tetrad (17,20) that locally diagonalizes the $T_{\mu \nu}$. It is therefore evident that $T_{\tau \rho} \epsilon^{\rho \nu} B_{\nu} g^{\tau \mu}$ lies on blade one, just by observing the expression (57), and using the extremal condition $\epsilon_{\mu \rho} * \epsilon^{\mu \lambda}=0$. Basically, because of the contractions $T_{(2) \rho} \epsilon^{\rho \nu} B_{\nu}=0$ and $T_{(3) \rho} \epsilon^{\rho \nu} B_{\nu}=0$. For similar reasons $T_{\tau \rho} * \epsilon^{\rho \nu} B_{\nu} g^{\tau \mu}$ lies on blade two, again because of the contractions $T_{(o) \rho} * \epsilon^{\rho \nu} B_{\nu}=0$ and $T_{(1) \rho} * \epsilon^{\rho \nu} B_{\nu}=0$. It is also clear that we could have chosen in place of (58,59) another pair of equations like,

$$
\begin{aligned}
& \left(T_{\tau \rho} * \epsilon^{\rho \nu} A_{\nu} g^{\tau \mu}\right)_{; \mu}=0 \\
& \left(T_{\tau \rho} \epsilon^{\rho \nu} A_{\nu} g^{\tau \mu}\right)_{; \mu}=0 .
\end{aligned}
$$

The conclusion is that we built through the imposed equations (58,59) two locally conserved currents. Even as important as that is that one lies on the local plane one, and the 
other on the local plane two as defined by the diagonalization at every point in spacetime of the Yang-Mills stress-energy tensor. Therefore these are local energy-momentum currents. The analysis on conserved charges and even a general treatment on conserved currents can be found in references $27-30$. It is now time to study the interaction of a source of non-Abelian and gravitational fields with an external agent also source of similar fields, and through perturbative techniques to prove the evolution of symmetries, as an evolution of local planes of Yang-Mills stress-energy diagonalization.

\section{FIRST ORDER PERTURBATIONS IN GEOMETRODYNAMICS}

We introduce first order perturbations to the relevant objects where $\varepsilon$ is an appropriate perturbative parameter,

$$
\begin{aligned}
& \tilde{g}_{\mu \nu}=g_{\mu \nu}+\varepsilon h_{\mu \nu} \\
& \tilde{\bar{\epsilon}}_{\mu \nu}=\bar{\epsilon}_{\mu \nu}+\varepsilon \bar{\omega}_{\mu \nu} \\
& \tilde{\epsilon}_{\mu \nu}=\epsilon_{\mu \nu}+\varepsilon \omega_{\mu \nu} .
\end{aligned}
$$

The perturbation objects $h_{\mu \nu}, \bar{\omega}_{\mu \nu}, \omega_{\mu \nu}$ and the one we are going to introduce next for the Yang-Mills tensor are of a physical nature caused by an external agent to the source of preexisting fields. It is worth stressing that they are not the result of a local first order coordinate transformation. They satisfy the perturbed Einstein-Maxwell-Yang-Mills vacuum field equations,

$$
\begin{aligned}
\tilde{R}_{\mu \nu} & =\tilde{T}_{\mu \nu}^{(y m)}+T_{\mu \nu}^{(\mathrm{em})} \\
f_{; \nu}^{\mu \nu} & =0 \\
* f_{; \nu}^{\mu \nu} & =0 \\
\tilde{f}_{\mid \nu}^{k \mu \nu} & =0 \\
* \tilde{f}_{\mid \nu}^{k \mu \nu} & =0 .
\end{aligned}
$$

We are focusing on the non-Abelian perturbations, therefore we are not introducing perturbations on Abelian electromagnetic fields, just for the purpose of solving the pure 
non-Abelian situation. However, in equations (66 67), or in the expression for $T_{\mu \nu}^{(e m)}$ we have to be aware of the perturbative nature of the metric tensor involved in these equations. We raise indices with the perturbed metric $\tilde{g}^{\mu \nu}=g^{\mu \nu}-\varepsilon h^{\mu \nu}$. We can write the perturbed block diagonalized Yang-Mills field strength projection in isospace, see equation (35), as,

$$
\tilde{\bar{f}}_{\mu \nu}=\tilde{A} \tilde{\bar{\epsilon}}_{\mu \nu}+\tilde{B} * \tilde{\bar{\epsilon}}_{\mu \nu}
$$

The perturbed $\tilde{\bar{f}}_{\mu \nu}$ is nothing but compact notation for $\operatorname{Tr}\left[\tilde{\vec{n}} \cdot \tilde{f}_{\mu \nu}\right]$. The perturbed local scalars $\tilde{A}$ and $\tilde{B}$ are not going to be explicitly involved in our analysis. Now, we move onto the extremal field associated to the diagonalization of the perturbed Yang-Mills stressenergy tensor, see equations (49) and (64). As it was done in references $\underline{13}, 15$ we impose the new condition,

$$
\tilde{\epsilon}_{\mu \nu} * \tilde{\epsilon}^{\mu \nu}=0
$$

and through the use of the identity (22), which is valid for every pair of antisymmetric tensors in a four-dimensional Lorentzian spacetime $\frac{15}{}$, when applied to the case $A_{\mu \alpha}=\tilde{\epsilon}_{\mu \alpha}$ and $B^{\nu \alpha}=* \tilde{\epsilon}^{\nu \alpha}$ yields the equivalent condition,

$$
\tilde{\epsilon}_{\mu \rho} * \tilde{\epsilon}^{\mu \lambda}=0
$$

We are brief in our presentation but care should be placed in our assertions. The long and explicit analysis would involve a perturbed version of the tensor (44) and a new duality transformation like in equations (49,50). It is at that point where the condition (71) is imposed, this time on the perturbed extremal field (64). In order to avoid confusions we highlight again that we are working with the perturbation to the extremal field associated to the stress-energy tensor diagonalization process, see equation (64). Even though we are developing a first order perturbative scheme, we would like to develop a general framework that conveys the ideas with more clarity, thus avoiding to write explicitly the first order approximations, specially in this section. Nonetheless we can display as an explicit example equation (172), that at first order can be written,

$$
\epsilon_{\mu \rho} * \epsilon^{\mu \lambda}+\varepsilon\left(\epsilon_{\mu \rho} * \omega^{\mu \lambda}+\omega_{\mu \rho} * \epsilon^{\mu \lambda}-\epsilon_{\mu \rho} * \epsilon_{\sigma \tau} h^{\mu \sigma} g^{\lambda \tau}-\epsilon_{\mu \rho} * \epsilon_{\sigma \tau} h^{\lambda \tau} g^{\mu \sigma}\right)=0
$$


We next proceed to write the four orthogonal vectors that are going to become an intermediate step in constructing the tetrad $\frac{18}{18}$ that diagonalizes the first order perturbed Yang-Mills stress-energy tensor,

$$
\begin{aligned}
& \tilde{S}_{(1)}^{\alpha}=\tilde{\epsilon}^{\alpha \lambda} \tilde{\epsilon}_{\rho \lambda} X^{\rho} \\
& \tilde{S}_{(2)}^{\alpha}=\sqrt{-\tilde{Q}_{y m} / 2} \tilde{\epsilon}^{\alpha \lambda} X_{\lambda} \\
& \tilde{S}_{(3)}^{\alpha}=\sqrt{-\tilde{Q}_{y m} / 2} * \tilde{\epsilon}^{\alpha \lambda} Y_{\lambda} \\
& \tilde{S}_{(4)}^{\alpha}=* \tilde{\epsilon}^{\alpha \lambda} * \tilde{\epsilon}_{\rho \lambda} Y^{\rho},
\end{aligned}
$$

It is evident that we are using now $\tilde{\epsilon}^{\mu \nu}$ in order to build the tetrad skeletons. Let us remember that this is the perturbed version (64) of the extremal field introduced in equation (49). In order to prove the orthogonality of the tetrad (74) it it is necessary to use the identity (22) for the case $A_{\mu \alpha}=\tilde{\epsilon}_{\mu \alpha}$ and $B^{\nu \alpha}=\tilde{\epsilon}^{\nu \alpha}$, that is,

$$
\tilde{\epsilon}_{\mu \alpha} \tilde{\epsilon}^{\nu \alpha}-* \tilde{\epsilon}_{\mu \alpha} * \tilde{\epsilon}^{\nu \alpha}=\frac{1}{2} \delta_{\mu}^{\nu} \tilde{Q}_{y m}
$$

where $\tilde{Q}_{y m}=\tilde{\epsilon}_{\mu \nu} \tilde{\epsilon}^{\mu \nu}$ is assumed not to be zero. We also need the condition (72). We are free to choose the vector fields $X^{\alpha}$ and $Y^{\alpha}$, as long as the four vector fields (74, 77) are not trivial. Let us remember under the present perturbative scheme that from all the possible normalized version of tetrads (74-77) that we called in the beginning of section II, $\tilde{W}_{(o)}^{\mu}, \tilde{W}_{(1)}^{\mu}, \tilde{W}_{(2)}^{\mu}, \tilde{W}_{(3)}^{\mu}$, we designated $\tilde{T}_{(o)}^{\mu}, \tilde{T}_{(1)}^{\mu}, \tilde{T}_{(2)}^{\mu}, \tilde{T}_{(3)}^{\mu}$ the particular perturbed tetrad that diagonalizes the local non-Abelian perturbed stress-energy tensor, see section $\amalg$ for the unperturbed scheme. As we did for the electromagnetic case in reference ${ }^{20}$ we just proved that we can reproduce for the perturbed fields a similar formalism and constructions put forward for the unperturbed fields. In particular, we are able to write our new local tetrad keeping the same local extremal skeleton structure as in the unperturbed case and define the new local planes of symmetry associated to the perturbed diagonalized Yang-Mills stressenergy tensor. The new local planes of symmetry are going to be tilted with respect to the unperturbed planes. As we see in the next section, the perturbed local conserved currents will stay continuously locked inside both local orthogonal perturbed planes as the evolution of the interaction takes place, satisfying the perturbed version of equations (58,59). They will continuously accompany the tilting of the local perturbed planes. 


\section{DYNAMICAL SYMMETRY BREAKING IN YANG-MILLS GEOMETRODYNAMICS}

We proceed then to write to first order the perturbed covariant derivative of a perturbed contravariant vector,

$$
\tilde{\nabla}_{\mu} \tilde{V}^{\lambda}=\frac{\partial \tilde{V}^{\lambda}}{\partial x^{\mu}}+\Gamma_{\mu \nu}^{\lambda} \tilde{V}^{\nu}+\varepsilon \tilde{\Gamma}_{\mu \nu}^{\lambda} V^{\nu}
$$

where the operator $\nabla$ indicates covariant derivative for notational convenience since we can write a tilde above it. The perturbed contravariant vector can be written $\tilde{V}^{\lambda}=V^{\lambda}+$ $\varepsilon V_{(1)}^{\lambda}$, where $V_{(1)}^{\lambda}$ is a local vector field. When we think of $V^{\lambda}$ in this manuscript, we will be thinking of the local currents $J^{\lambda}$. It is worth stressing again that we are studying genuine physical perturbations to the gravitational and non-Abelian fields by external agents to the preexisting source. We are not introducing first order coordinate transformations of the kind $\tilde{x}^{\alpha}=x^{\alpha}+\varepsilon \zeta^{\alpha}$, where the local vector field $\zeta^{\alpha}\left(x^{\sigma}\right)$ is associated to a first order infinitesimal local coordinate transformation scheme $\mathrm{w}^{\underline{16}}$. We proceed next to write the first order perturbed covariant derivative of a first order perturbed local contravariant current vector,

$$
\tilde{\nabla}_{\mu} \tilde{J}^{\lambda}=\frac{\partial \tilde{J}^{\lambda}}{\partial x^{\mu}}+\Gamma_{\mu \nu}^{\lambda} \tilde{J}^{\nu}+\varepsilon \tilde{\Gamma}_{\mu \nu}^{\lambda} J^{\nu}
$$

Following the literature in perturbative schemes, see $\underline{16} \underline{\underline{31}}-\underline{\underline{34}}$ and references therein as examples, we can write the first order perturbed affine connection as,

$$
\tilde{\Gamma}_{\mu \nu}^{\lambda}=\frac{1}{2} g^{\lambda \sigma}\left(h_{\mu \sigma ; \nu}+h_{\nu \sigma ; \mu}-h_{\mu \nu ; \sigma}\right),
$$

where the covariant derivatives in (81) are calculated with the unperturbed affine connection. The local perturbed currents introduced in equation (80) are the currents that satisfy the perturbed version of equations (58/59), that is,

$$
\begin{aligned}
& \left(\begin{array}{lll}
\tilde{T}_{\tau \rho} * \tilde{\epsilon}^{\rho \nu} & \tilde{B}_{\nu} & \left.\tilde{g}^{\tau \mu}\right)_{; \mu}=0
\end{array}\right. \\
& \left(\begin{array}{lll}
\tilde{T}_{\tau \rho} & \tilde{\epsilon}^{\rho \nu} \tilde{B}_{\nu} & \left.\tilde{g}^{\tau \mu}\right)_{; \mu}=0 .
\end{array}\right.
\end{aligned}
$$


Let us not forget that in equations $(82,83)$ the metric tensor is perturbed like in equation (62). We can rewrite equation (80) as follows,

$$
\tilde{\nabla}_{\mu} \tilde{J}^{\lambda}=\nabla_{\mu} J^{\lambda}+\varepsilon \frac{\partial J_{(1)}^{\lambda}}{\partial x^{\mu}}+\varepsilon \Gamma_{\mu \nu}^{\lambda} J_{(1)}^{\nu}+\varepsilon \tilde{\Gamma}_{\mu \nu}^{\lambda} J^{\nu}
$$

where we have written the first order perturbed local current as $\tilde{J}^{\lambda}=J^{\lambda}+\varepsilon J_{(1)}^{\lambda}$. As we did in a previous work $^{20}$, we can imagine spacetime between an initial constant time hypersurface and an intermediate constant time hypersurface, right when the perturbation starts taking place, a region of spacetime where the unperturbed local currents are considered to be conserved, that is $\nabla_{\mu} J^{\mu}=0$. In this initial region of spacetime, the unperturbed local currents, lie inside the unperturbed local blade one or blade two, the local planes of gauge symmetry. Now, the original local current $J^{\mu}$ will be conserved no longer in the spacetime region determined by the intermediate constant time hypersurface and a final constant time hypersurface. After the intermediate constant time hypersurface, the perturbation generated by the external agent to the source starts taking place and the ensuing conservation equation will be for the perturbed local current $\tilde{\nabla}_{\mu} \tilde{J}^{\mu}=0$. The geometrical reason for this can be associated to the fact that the local planes of symmetry, both blade one and two, will be tilted by the perturbation with respect to the planes on the initial field structure. There will be at every point in spacetime new local planes of symmetry. This geometrical effect can be visualized through the new perturbed $\tilde{T}_{(o)}^{\mu}, \tilde{T}_{(1)}^{\mu}, \tilde{T}_{(2)}^{\mu}, \tilde{T}_{(3)}^{\mu}$, the particular tetrad that diagonalizes the local stress-energy $\tilde{T}_{\mu \nu}$, as we can see from the perturbed version of expression (57),

$$
\tilde{T}_{\mu \nu}=\left[-\tilde{C} \tilde{T}_{(o) \mu} \tilde{T}_{(o) \nu}+\tilde{D} \tilde{T}_{(1) \mu} \tilde{T}_{(1) \nu}+\tilde{E} \tilde{T}_{(2) \mu} \tilde{T}_{(2) \nu}+\tilde{F} \tilde{T}_{(3) \mu} \tilde{T}_{(3) \nu}\right],
$$

The new local planes or blades of symmetry in spacetime after the perturbation took place, will no longer coincide with the old ones. This is the reason why after the perturbations already took place the equation $\nabla_{\mu} J^{\mu}=0$ is no longer valid and according to equation (84) the following result will be valid,

$$
\nabla_{\mu} J^{\lambda}=-\varepsilon \frac{\partial J_{(1)}^{\lambda}}{\partial x^{\mu}}-\varepsilon \Gamma_{\mu \nu}^{\lambda} J_{(1)}^{\nu}-\varepsilon \tilde{\Gamma}_{\mu \nu}^{\lambda} J^{\nu}
$$


This is exactly what we might call dynamic symmetry breaking in non-Abelian YangMills geometrodynamics. The old currents $J^{\lambda}$ will be no longer conserved, only the new ones $\tilde{J}^{\lambda}$ will be.

\section{CONCLUSIONS}

As in the Abelian case ${ }^{20}$ the evolution of symmetries resides in the evolution of local planes of symmetry. The local planes of symmetry are associated to the local diagonalization of the Yang-Mills stress-energy. In the non-Abelian field strength we have a local isospace projection by a unit vector plus the two unit local vectors that intervene in the process of the block diagonalization of the non-Abelian field strength. These vectors provide two more local scalars to solve the additional problem of finding local conserved currents in addition to the planes that diagonalize the Yang-Mills stress-energy tensor. We find simultaneously both, the local planes that diagonalize the stress-energy and the two local conserved currents, one for each local plane. We also block diagonalize the non-Abelian field strength. As the interaction between the external agent and the source evolves, the loss of symmetry is visualized through the tilting of the local planes that diagonalize the stress-energy. The local currents that on an intermediate hypersurface lied each on one of the initial intermediate planes, as the interaction takes place are also going to tilt. They evolve by staying on the new perturbed and tilted planes, getting themselves tilted as well. It is as if they are locked inside the local planes of symmetry, one on each plane. The initial symmetry is lost. As the interaction proceeds, the planes of symmetry tilt and new symmetries arise, continuously. The five local isospace unit vectors involved in the analysis, that is, $\vec{n}, \vec{m}, \vec{l}, \vec{p}, \vec{q}$, rotate in the unit isosurface as the evolution takes place because they are also perturbed. This is the four-dimensional local Lorentzian expression of what we might call, dynamic symmetry breaking in the non-Abelian Yang-Mills case. There is no mass generation as in the schemes put forward in both the Quantum Field dynamic symmetry breaking or the Quantum Field Higgs mechanism. Instead there is a generation of curvature. A curvature that is associated to the dynamical interaction between the perturbing agent and the source. We quote from ${ }^{35}$ "With the advent of special and general relativity, the symmetry laws gained new importance. Their connection with the dynamic laws of physics takes on a much more integrated and interdependent relationship than in classical mechanics, where logically 
the symmetry laws were only consequences of the dynamical laws that by chance possess the symmetries. Also in the relativity theories the realm of the symmetry laws was greatly enriched to include invariances that were by no means apparent from daily experience. Their validity rather was deduced from, or was later confirmed by complicated experimentation. Let me emphasize that the conceptual simplicity and intrinsic beauty of the symmetries that so evolve from complex experiments are for the physicists great sources of encouragement. One learns to hope that Nature possesses an order that one may aspire to comprehend."

\section{ACKNOWLEDGMENTS}

I would like to thank Professor D. Singleton for pointing out to the author the existence of the manuscript by N. Cabibbo and E. Ferrari.

\section{REFERENCES}

${ }^{1}$ Y. Nambu and G. Jonna-Lasinio, Phys. Rev. 122, 345 (1961).

${ }^{2}$ J. Schwinger, Phys. Rev. 125, 397 (1962); Phys. Rev. 128, 2425 (1962).

${ }^{3}$ R. Jackiw and K. Johnson, Phys. Rev. D 8, 2386 (1973).

${ }^{4}$ J. M. Cornwall and R. E. Norton, Phys. Rev. D 8, 3338 (1973).

${ }^{5}$ D. J. Gross and A. Neveu, Phys. Rev. D 10, 3235 (1974).

${ }^{6}$ E. J. Eichten and F. L. Feinberg, Phys. Rev. D 10, 3254 (1974).

${ }^{7}$ S. Coleman, R. Jackiw and H. D. Politzer, Phys. Rev. D 10, 2491 (1974).

${ }^{8}$ J. M. Cornwall, R. Jackiw and E. Tomboulis, Phys. Rev. D 10, 2438 (1975).

${ }^{9}$ E. C. Poggio, E. Tomboulis and S. H. H. Tye, Phys. Rev. D 11, 2839 (1975).

${ }^{10}$ K. Lane, Phys. Rev. D 10, 1353 (1974).

${ }^{11}$ S. Weinberg, Phys. Rev. D 13, 974 (1976).

${ }^{12}$ P. W. Higgs, Phys. Lett. 13, 508 (1964); Phys. Lett. 12, 132 (1965); Phys. Rev. 145, 1156 (1966); F. Englert and R. Brout, Phys. Rev. Lett. 13, 321 (1964); G. S. Guralnik, C. R. Hagen and T. W. B. Kibble, Phys. Rev. Lett. 13, 585 (1964); T. W. Kibble, Phys. Rev. 155, 1554 (1967) .

${ }^{13}$ A. Garat, J. Math. Phys. 46, 102502 (2005). 
${ }^{14}$ J. A. Schouten, Ricci Calculus: An Introduction to Tensor Calculus and Its Geometrical Applications (Springer, Berlin, 1954).

${ }^{15}$ C. Misner and J. A. Wheeler, Annals of Physics 2, 525 (1957).

${ }^{16}$ S. Weinberg, Gravitation and Cosmology (John Wiley, 1972).

${ }^{17}$ C. W. Misner, K. S. Thorne, and J. A. Wheeler, Gravitation (W. H. Freeman, San Francisco, 1973).

${ }^{18}$ A. Garat, arXiv:1306.2174.

${ }^{19}$ A. Garat, gr-qc/0602049.

${ }^{20}$ A. Garat, arXiv:1306.0602.

${ }^{21}$ N. Cabibbo and E. Ferrari, Nuovo Cim. 23, 1147 (1962).

${ }^{22}$ M. Carmeli, Classical Fields: General Relativity and Gauge Theory (J. Wiley \& Sons, New York, 1982).

${ }^{23}$ C. N. Yang and R. L. Mills, Phys. Rev. 96, 191 (1954).

${ }^{24}$ R. Utiyama, Phys. Rev., 101, 1597 (1956).

${ }^{25}$ Y. Choquet-Bruhat and C. DeWitt-Morette, Analysis, Manifolds and Physics (Elsevier Science Publishers B.V., 1987).

${ }^{26} \mathrm{~W}$. Greiner and B. Mueller, Quantum Mechanics, Symmetries (Springer Verlag Gmbh, 1989).

${ }^{27}$ R. Lazkoz, J. M. M. Senovilla and R. Vera, Class. Quant. Grav. 20, 4135 (2003).

${ }^{28}$ G. Bergqvist, I. Eriksson and J. M. M. Senovilla, Class. Quant. Grav. 20, 2663 (2003).

${ }^{29}$ J. M. M. Senovilla, General Electric-Magnetic decomposition of fields, positivity and Rainich-like conditions (Plenary talk given at the Spanish Relativity Meeting published in the proceedings of ERE-00, 2000).

${ }^{30}$ J. M. M. Senovilla, Class. Quant. Grav. 17, 2799 (2000).

${ }^{31}$ L. Papantonopoulos Physics of Black Holes (Springer, 2009).

${ }^{32}$ T. Regge and J.A. Wheeler, Phys. Rev. 108, 1063 (1957).

${ }^{33}$ V. Moncrief, Annals Phys. 88, 323 (1974).

${ }^{34}$ A. Garat and R. Price, Phys. Rev. D, 61, 044006 (2000).

${ }^{35}$ C. N. Yang, Nobel Lectures, Physics (Elsevier Publishing Company, Amsterdam, 1964). 